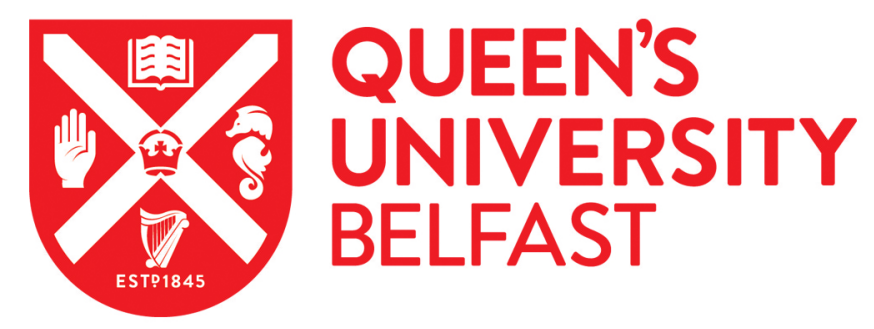

\title{
Effect of turbulent dispersion on hydrodynamic characteristics in a liquid jet ejector
}

Sharma, D., Patwardhan, A., \& Ranade, V. (2018). Effect of turbulent dispersion on hydrodynamic characteristics in a liquid jet ejector. Energy, 164, 10-20. https://doi.org/10.1016/j.energy.2018.08.171

\author{
Published in: \\ Energy
}

\section{Document Version:}

Peer reviewed version

Queen's University Belfast - Research Portal:

Link to publication record in Queen's University Belfast Research Portal

\section{Publisher rights}

Copyright 2018 Elsevier.

This manuscript is distributed under a Creative Commons Attribution-NonCommercial-NoDerivs License

(https://creativecommons.org/licenses/by-nc-nd/4.0/), which permits distribution and reproduction for non-commercial purposes, provided the author and source are cited.

\section{General rights}

Copyright for the publications made accessible via the Queen's University Belfast Research Portal is retained by the author(s) and / or other copyright owners and it is a condition of accessing these publications that users recognise and abide by the legal requirements associated with these rights.

Take down policy

The Research Portal is Queen's institutional repository that provides access to Queen's research output. Every effort has been made to ensure that content in the Research Portal does not infringe any person's rights, or applicable UK laws. If you discover content in the Research Portal that you believe breaches copyright or violates any law, please contact openaccess@qub.ac.uk. 
Effect of Turbulent Dispersion on Hydrodynamic Characteristics in a Liquid Jet Ejector

Deepankar Sharma ${ }^{1,2}$, Ashwin Patwardhan ${ }^{2}$ and Vivek Ranade ${ }^{1,3}$

${ }^{1}$ Chemical Engineering Division,

CSIR - National Chemical Laboratory, Pune 411008

${ }^{2}$ Institute of Chemical Technology, Mumbai 400019

${ }^{3}$ School of Chemistry and Chemical Engineering

Queen's University Belfast, Belfast, NI, UK

Corresponding author: V.Ranade@qub.ac.uk

Key words: CFD, Gas Induction, Gas - liquid ejectors, turbulent dispersion, multiphase, ejectors 


\begin{abstract}
$\underline{\text { Abstract }}$
Gas induction using a liquid jet is an extensively studied phenomenon. Many studies have been published detailing various hydrodynamic and mass transport aspects of gas induction in ejectors. Conversely, multiple studies have been published detailing jet dynamics, jet break up, nozzle geometry, effects of turbulence etc. In the modelling of the jet ejector systems CFD framework is used as the flow of gas is coupled with the liquid flow and the flow parameters like volume fraction, pressure drop/profile and velocity profiles both local and average are highly interdependent. Hence, it becomes important to capture all the flow physics in the simulations in order to understand the hydrodynamics. In the previous models the effect of turbulent dispersion was not included which led to incorrect calculation of phase profile, and other related parametric values. In the present work, the turbulence effects and its impact on the jet dynamics and gas induction rate in an ejector have been studied. Capturing the correct phase as well as velocity profile is imperative for the extension of the model to mass transfer and reactions. Lastly, other hydrodynamic properties like pressure drop, phase hold up and pressure profile help better the design correlations for jet ejectors.
\end{abstract}




\section{Introduction}

Gas-liquid ejectors predominantly, are used in fast gas liquid reactions like hydrogenation, chlorination etc. First of these devises was put into practice in 1925 as a steam ejector and over the years ejectors have found use in various chemical and physical processes [1]. The driving force for gas induction is the momentum exchange between the fast moving jet of the primary fluid (usually liquid) and the surrounding secondary phase (gas/liquid). Gas induction shows a definite correlation with various geometric parameters of the ejector like the nozzle diameter, diffuser angle, mixing tube diameter and length. Due to the turbulent nature of the jet $(\operatorname{Re}>100000)$, the gas and liquid mix together to form a fairly homogeneous mixture causing very high mass transfer rates in gas-liquid ejectors ${ }^{[2,3,4]}$.

Experimental studies have reported mixing of gas and liquid inside the mixing tube region ${ }^{[5,6]}$. Witte ${ }^{[7]}$ calls this phenomenon a 'mixing shock' and describes it as an abrupt mixing of phase resulting in a homogeneous bubbly flow along the axis of ejector. Witte further explains, this 'shock' is caused by the difference in velocities between the two phases. Due to the high shear forces acting at the gas-liquid interface the phases mix together to increase the area of contact and hence achieving a lower surface energy state by the creation of new surface. This explanation of mixing shock is on the similar lines to Rayleigh instability where one fluid which is moving faster than the other tend to mix together near the phase interphase. However, in the case of gas-liquid ejectors the secondary fluid motion is caused by the primary fluid motion due to momentum exchange and is not forced. Umoto et al ${ }^{[8]}$ describes the mixing of phases as a dispersion along the length of the jet instead of a sudden 'shock' or a disconinuity. In the present work, the second hypothesis is explored as the as expansion of jet was experimentally observed to be gradual. Furthermore, the term 'shock' suggests a discontinuity which cannot be modelled using differential equations used in the CFD framework.

At a low jet velocity, completely stratified flow was reported by Ben Brahim et al [9] and $\mathrm{Bin}{ }^{[10]}$. As the velocity was increased the flow regime transitions from stratified to dispersed flow. This transition may be due to the change in the regime of flow from laminar to turbulent due to which there is an increase in the expansion of the jet as well as break up of jet. This phenomenon was studied by Kitamura and Takahashi ${ }^{[11]}$ and it was found that the turbulence, breakup of jet and jet expansion are correlated. As the turbulence in the free falling jet increases, the motion of eddies along with the surface tension forces at the gas-liquid interface increase surface imperfections. This leads to chipping of jet and formation of a region 
around the jet where the primary and surrounding fluids mix with each other. After a point the jet continuity breaks and air and water flow as a mixture. The jet turbulence at the point of formation of the free falling jet depends upon geometry of the nozzle, For example a jet created using a long cylindrical tube will have different expansion coefficient and breakup length as compared to a jet created using a converging nozzle. Hence, from the above stated experimental observations of Bin and Kitamura and Takahashi ${ }^{[10,11]}$, it can be concluded that turbulence effects play a very important role in the jet hydrodynamics.

Shinijo and Umemura ${ }^{[16]}$ performed Direct Numerical Simulations (DNS) using VOF (volume of fluid) formulation on a downward flowing jet and plotted the surface of the jet. The simulations were performed on a very small jet diameter over a limited length. Similar attempt was made to simulate jet breakup using LES methodology by Xiao et al. ${ }^{[17]}$ Experiments show that the jet surface after leaving the nozzle becomes unstable and starts the chip away leading to primary breakup of the jet. However, the region of the jet simulated by the above described models is very limited, as the LES and DNS simulations are computationally expensive. The requirement of computational resources for the simulation of an entire ejector geometry is very large if one were to capture the effects of such anisotropic turbulence. Hence, in order to get meaningful results turbulent effects must incorporated using a turbulence model. Many such studies have been described in the published literature and are discussed below.

Kandakure et al. ${ }^{[12]}$ and Kim et al. ${ }^{[13]}$ developed CFD methodologies capable of predicting gas induction in down flow and horizontal gas-liquid ejectors respectively. Both these methodologies use the two-phase Mixture model ${ }^{[14]}$ approach in which the volumetric drag source is modelled as the resulting slip velocity attained as the two fluids are assumed to be at equilibrium. A single momentum equation model was selected because the jet and the surrounding air move together and coaxially along the axis of the ejector. Secondly, to avoid resolution of the complex interaction between the jet surface and surrounding air a general slip velocity formulation was used. As the motive of the aforementioned works was the calculation of gas induction in jet ejectors the described methodology was successful. While Kandakure et al. used percentage slip as a fitting parameter and Kim et al. used drag force formulation using bubble diameter as a fitting parameter. However, the phase profile predicted was completely stratified with no mixing whatsoever, the change in diameter of the jet is less than $5 \%$, which is not consistent with the e xperimental observations. 
Sharma et al. ${ }^{[20]}$ used single phase model to simulate gas induction. This model used a fitting parameter to correlate the secondary liquid phase induction to the gas induction found experimentally. The main finding was that the gas and liquid induction exhibit the same trend which only depends on the geometry of the ejector. The second finding was that the size of the nozzle has the strongest impact on the gas induction. The paper further shows that the fitting parameter or the measure of gas liquid interaction is strongly influenced by the ratio of the diameter of nozzle to the throat of the ejector. This strengthens the claim that the jet dynamics plays an important role in the overall performance of the ejector.

Umoto et al. ${ }^{[8]}$ and Yadav ${ }^{[15]}$ developed a different approach using Euler-Euler framework. Instead of slip velocity the momentum exchange was modelled using drag law formulation. The data presented by Yadav ${ }^{[15]}$ shows that by adjusting the bubble diameter as a parameter, the simulated gas induction can be fitted to experimental values. Whereas, results presented by Umoto et al. ${ }^{[8]}$ use the same Euler - Euler framework do show phase mixing. However, there is absolutely no description of any specific model changes, boundary condition, model constants etc. These simulations were done using Star CCD commercial CFD software.

In the present work, it is hypothesized that the mixing and breakup of the jet is caused due to turbulence and can be captured by representing this appropriately in the model. The EE modelling approach is further explored as it is relatively less expensive computationally. The objective of the work is to develop a methodology using the current CFD framework that not only predict the gas induction rate in a gas-liquid ejector but also predict the proper flow development inside the ejector. Since, no data is available on the phase profile or the velocity profile of gas and liquid inside the jet ejector there is no way for validating the model. However, a quantitative discussion on the flow profile is possible. This framework may be helpful in the development of mass transfer models between gas and liquid phases and may lead to further development of detailed models that may incorporate anisotropic turbulence modelling that may consider effects of regional turbulence.

\section{Mathematical Model}

The two fluid Euler-Euler framework for solving multiphase flow was adopted in which the secondary phase dispersed within the continuous/primary phase. The dispersed phase is treated like a continuum as both the phases in the EE approach are assumed to be interpenetrating. The motion of the volume fraction of the dispersed phase is calculated by solving 
the dispersed phase continuity equation. The interactive forces between the two phases are calculated separately and added to the momentum equation as a volumetric momentum source. Quantities like density, interactive forces, stresses etc. are calculated with the help of the volume fraction of the fluid. The final equations were derived using Reynolds Averaging (RANS) method.

The volume averaged governing equations for mass (continuity) and momentum and described in Equations (1) and (2). Equation (1) and (2) represent the generalized format of Navier - Stokes equation. Where the vector quantity $\vec{u}_{k}$ represents the instantaneous velocity vector of the fluid. These equations are valid for laminar as well as turbulent flows. However, in the present work RANS methodology for modelling turbulent flows is used, Equations (1) and (2) were averaged to formulate equations (3) and (4). For the sake of brevity, the averaging procedure and the related laws for averaging are kept out of the discussion of this paper. One may find the details of the RANS in the Ranade ${ }^{[18,24]}$ and Ranade and Van den Akker ${ }^{[25] .}$

$$
\begin{aligned}
& \frac{\partial\left(\alpha_{k} \rho_{k}\right)}{\partial t}+\nabla \cdot\left(\alpha_{k} \rho_{k} \vec{u}_{k}\right)=\sum_{p=1, p \neq k}^{n} S_{p k} \\
& \frac{\partial\left(\alpha_{k} \rho_{k} \vec{u}_{k}\right)}{\partial t}+\nabla \cdot\left(\alpha_{k} \rho_{k} \vec{u}_{k} \vec{u}_{k}\right)=-\alpha_{k} \nabla p-\nabla \cdot\left(\alpha_{k} \overline{\overline{\tau_{k}}}\right)+\alpha_{k} \rho_{k} \vec{g}_{k}+\vec{F}_{k}
\end{aligned}
$$

The RANS equation pertaining to the solution are given in Equations (3) and (4). These equations represent the two fluid, 2-dimensional modelling approach which is relevant to the system under study. In Equation (3) and (4) the averaged quantities (overhead bar represents the averaged quantity) represent the additional momentum and mass motion terms because of turbulence that govern the overall motion of the phase $\mathrm{k}$. The $u_{k, i}^{\prime}$ term represents the fluctuation portion of the overall velocity $\vec{u}_{k}$ of phase $\mathrm{k}$ in the ith direction. Whereas $U_{k, i}$ represent the mean velocity of phase $\mathrm{k}$ in direction $\mathrm{i}$.

$$
\begin{aligned}
& \frac{\partial\left(\alpha_{k} \rho_{k}\right)}{\partial t}+\frac{\partial}{\partial x_{j}}\left(\alpha_{k} \rho_{k} U_{k, j}+\rho_{k} \overline{\alpha_{k}^{\prime} u_{k, j}^{\prime}}\right)=0 \\
& \frac{\partial\left(\alpha_{k} \rho_{k} U_{k, i}+\overline{\alpha_{k}^{\prime} u_{k, i}^{\prime}}\right)}{\partial t}+\frac{\partial}{\partial x_{j}}\left(\alpha_{k} \rho_{k} U_{k, i} U_{k, j}\right)=-\alpha_{k} \frac{\partial p}{\partial x_{i}}-\overline{\alpha_{k}^{\prime} \frac{\partial p}{\partial x_{i}}}-\frac{\partial}{\partial x_{j}}\left(\alpha_{k} \tau_{k, i j}\right) \\
& +\alpha_{k} \rho_{k} g_{k, i}+\bar{F}_{k, i}-\frac{\partial}{\partial x_{j}}\left(\rho_{k} \alpha_{k} \overline{u_{k, i}^{\prime} u_{k, j}^{\prime}}+U_{k, i} \overline{\alpha_{k}^{\prime} u_{k, j}^{\prime}}+U_{k, j} \overline{\alpha_{k}^{\prime} u_{k, i}^{\prime}}\right)
\end{aligned}
$$


To solve the RANS equations closure models are required for modelling the Reynolds stresses. The Reynolds stresses appearing in the derived RANS Equation (4) were modelled like viscous stresses by employing Boussinesq hypothesis, with the coefficient of turbulent momentum transfer called turbulent viscosity. In the Boussinesq hypothesis the overall motion caused by the fluctuating anisotropic behaviour of the eddy motion is modelled using an isotropic diffusion term as shown in Equation (5). Approximations derived by Johansen ${ }^{[19,24}$ ${ }^{25]}$ as seen in Equation (6), were used to further simplify the RANS equations for momentum transfer (Equation (4)). Furthermore, third or higher order terms like $\overline{\alpha_{k}^{\prime} u_{k, i}^{\prime} u_{k, j}^{\prime}}$ and

$$
\begin{aligned}
& \frac{\partial}{\partial x_{j}}\left(\overline{\alpha_{k}^{\prime} \frac{\partial}{\partial x_{j}}\left(u_{k, j}^{\prime}\right)}\right) \text { etc. were neglected. } \\
& \overline{\rho_{k} \overline{u_{k, i}^{\prime} u_{k, j}^{\prime}}}=-\mu_{t}\left(\frac{\partial U_{k, i}}{\partial x_{j}}+\frac{\partial U_{k, j}}{\partial x_{i}}\right)+\frac{2}{3} k \delta_{i j} \\
& \overline{\alpha_{k}^{\prime} \frac{\partial p}{\partial x_{i}}}=-\rho_{k} \overline{\alpha_{k}^{\prime} u_{k, j}^{\prime}} \frac{\partial U_{k, i}}{\partial x_{i}}-\rho_{k} U_{k, j} \frac{\partial}{\partial x_{i}} \overline{\alpha_{k}^{\prime} u_{k, i}^{\prime}}
\end{aligned}
$$

Multiple closure models are available for modelling $\mu_{t}$ appearing in Equation (5), these models are discussed in the later part of this section. One RANS term, as shown in equation (3), also appears in the equation of continuity. This term is added as a source term in the continuity equation solution as a turbulent dispersion force. The turbulent dispersion effects were implemented using Equation (7). As seen from the equation that the inherent fluctuations due to the turbulent behaviour cause dispersion and by extension mixing among the phases. This equation was implemented as a source term in the secondary phase continuity equation and solved alongside the model. This implementation adds one more parameter to the model; turbulent dispersion Schmidt number represented by $\sigma_{\mathrm{k}}$. This parameter controls the overall coefficient of dispersion of the fluid which is $\left(v_{T} / \sigma_{G}\right)$. This is an extension of the Boussinesq hypothesis where phenomenon such as surface chipping and eddy motion caused by turbulence is modelled as effective diffusion.

$$
\overline{u_{k i}^{\prime} \alpha_{k i}^{\prime}}=-\frac{v_{t}}{\sigma_{G}}\left(\frac{\partial \alpha_{k}}{\partial x_{i}}\right)
$$


The interfacial exchange terms play a very important role in a gas ejector as a falling water jet causes the surrounding air to flow along with it. In other words the air flow is caused by the momentum/energy transferred across the interface. The interface momentum exchange, denoted by $\overline{F_{k}}$ in Equation (2) \& (4), between the two phases depends upon velocity difference between the phases (slip velocity) as shown in the generic interface force Equation (8). There are three major kinds of interface exchange forces namely drag, lift and virtual mass forces. Considering that fluid dynamics of ejectors is dictated by liquid phase momentum which is to say that the gas is basically induced into the liquid jet and flow co-axially. Under such conditions the virtual mass force and lift force terms were not significant compared to the drag force and were therefore neglected in the present work ${ }^{[12,15]}$. The drag force is exerted at the interface of any two phases and is considered in the model. The generic expression of the volumetric drag force in the $\mathrm{i}^{\text {th }}$ direction can be seen in Equation (8), this formulation inherently satisfies the force balance between the phases.

In the formulation of drag force the drag coefficient $C_{D}$ is used which may change according to the shape of the bubble/drop of the dispersed phase. The choice of the suitable model for simulation of drag depends primarily upon flow physics, hold up, size and shape of the bubble. Chanson ${ }^{[21]}$ and Witte ${ }^{[7]}$ state that the flow in an ejector is highly dispersed and almost homogeneous. Chanson further suggests that the jet near the nozzle contains air bubbles as small as $10 \mu \mathrm{m}$. Furthermore, Witte calls the dispersion in the mixing tube region as a very fine dispersion of air bubbles in water. Hence, from these experimental observation it may be concluded that the bubbles present in the jet as well as the mixture are small in diameter. Small bubbles may be modelled as hard spheres. While the bubble diameter will be different at various regions of the ejector the model uses single bubble diameter to calculate the overall drag force between the two phases. Given these postulations Schiller-Naumann ${ }^{[12,13,15]}$ drag coefficient, described in Equation (10), was used to model the interfacial forces between the two phases as this model is suited for highly turbulent flow with fine dispersion of bubbles. Hence, now there are two input parameters in the model first being bubble diameter and second being the turbulent Schmidt number. The impact of both these parameters is analysed in the next section.

$$
\vec{F}_{k}=\sum_{q=1}^{n} K_{k q}\left(\vec{U}_{q}-\vec{U}_{k}\right)
$$




$$
\begin{gathered}
\bar{F}_{q, i}=-\frac{3 \alpha_{p} \alpha_{q} \rho_{p} C_{D}\left(\sum_{j}\left(U_{q, j}-U_{p, j}\right)^{2}\right)^{0.5}\left(U_{q, i}-U_{p, i}\right)}{4 d_{B}} \\
C_{D}=\left\{\begin{array}{cc}
24 / \operatorname{Re}\left(1+0.15 \operatorname{Re}^{0.687}\right) & \operatorname{Re}<1000 \\
0.44 & \operatorname{Re} \geq 1000
\end{array}\right.
\end{gathered}
$$

To solve the RANS equations closure models are necessary order to solve the system of equations. Two equation Standard k- $\varepsilon$ (Ske) model was used to model the turbulent viscosity that appeared as a closure model parameter in Equations (5) and (7). Ske model was solved for the gas-liquid mixture and properties like density and mixture velocity were calculated using volume averaging of density and momentum. The standard equations for mixture standard $\mathrm{k}-\varepsilon$ model are provided below. The selection of Ske is predicated upon multiple factors firsts of which is the fact that all the major phenomena/forces are acting in the bulk of the flow and not at the boundary where other turbulent models like k- $\omega / \mathrm{k}-\omega$ SST excel. As there is no swirl/vortex flow in the ejector, hence, the use of RSM models is not necessary. Among the $\mathrm{k}-\varepsilon$ models, one of the major documented drawbacks of the Ske model is the over prediction of turbulent viscosity at $(\operatorname{Re}<10,000)$ lower Reynolds numbers. However, in the current situation the Reynolds number is very high $(\operatorname{Re}>100,000)$. Considering the complexity of turbulent two phase flows in gas-liquid ejectors considered in this work and lack of experimental data to critically evaluate performance of turbulence models, we have used relatively simple and robust Ske model to perform simulations of the jet ejector system. ${ }^{[22,23 \text {, }}$ $18]$

$$
\begin{aligned}
& \mu_{T}=\frac{C_{\mu} \rho_{m} k^{2}}{\varepsilon} \\
& \frac{\partial\left(\rho_{m} k\right)}{\partial t}+\frac{\partial\left(\rho_{m} U_{m, i} k\right)}{\partial x_{i}}=\frac{\partial}{\partial x_{i}}\left(\frac{\mu_{T}}{\sigma_{k}} \frac{\partial k}{\partial x_{i}}\right)+G-\rho_{m} \varepsilon \\
& \frac{\partial\left(\rho_{m} \varepsilon\right)}{\partial t}+\frac{\partial\left(\rho_{m} U_{m, i} \varepsilon\right)}{\partial x_{i}}=\frac{\partial}{\partial x_{i}}\left(\frac{\mu_{T}}{\sigma_{\varepsilon}} \frac{\partial \varepsilon}{\partial x_{i}}\right)+\frac{\varepsilon}{k}\left(C_{1} G-C_{2} \rho_{m} \varepsilon\right) \\
& \rho_{m}=\sum_{i=1}^{n} \alpha_{i} \rho_{i}
\end{aligned}
$$




$$
U_{m, i}=\frac{\sum_{k=1}^{n} \alpha_{k} \rho_{k} U_{k, i}}{\sum_{k=1}^{n} \alpha_{k} \rho_{k}}
$$

The ejector geometry detailing the dimensions shown in Figure 1 was modelled using axi-symmetric approximation and the gas inlet was modelled as a slit of equivalent area. The primary nozzle diameter is $8 \mathrm{~mm}\left(\mathrm{D}_{\mathrm{N}}\right)$ and the ejector throat diameter is $16 \mathrm{~mm}\left(\mathrm{D}_{\mathrm{T}}\right)$. The length of the ejector is $128 \mathrm{~mm}$ or $\left(8 \mathrm{D}_{\mathrm{T}}\right)$. The length of converging section is $80 \mathrm{~mm}$ and the diverging section is $200 \mathrm{~mm}$. The diverging angle is 5 and the converging angle is $12^{\circ}$. Water was used as the motive fluid and air was used as the secondary fluid with a constant density (1.221 $\mathrm{kg} / \mathrm{m}^{3}$ ). The ejector is oriented in down-flow fashion and where water enters from the top liquid nozzle and exits from the bottom, gravitational force acts along the axis of the ejector. As the volumetric flow rates of water through the nozzle were known, the inlet velocity was used as the boundary condition for primary liquid. Pressure at suction chamber inlet was set at $0 \mathrm{~Pa}$ gauge pressure. The ejector outlet pressure was also set at $0 \mathrm{~Pa}$ gauge pressure. The no-slip adiabatic boundary condition was enforced at the walls of the ejector. Table 1 lists the boundary conditions and the values used to simulate the geometry described in Figure 1. Table 2 lists the closure models used for the solution.

The boundary conditions for $\mathrm{k}$ and $\varepsilon$ were specified by specifying the turbulent intensity and hydraulic diameter. The value of turbulent intensity was kept at $5 \%$ which corresponds to a medium bounded turbulent flow intensity. It was found that an increase in the turbulent intensity at the inlet increases the overall jet dispersion and shortens the jet breakup length. Which is expected as increase in the turbulent intensity directly results in higher turbulent viscosity which in turn increases the dispersion coefficient as shown in Equations (16)-(18). However, the turbulent intensity at the inlet for the ejector domain is a result of flow up-steam, from the pump to the nozzle, and is dependent upon the pipe geometry connecting the pump to the nozzle like number of bends, discharge coefficients of various valves, inline flow meters etc. Hence the boundary condition will be unique for each system and, to make a generalized model, the role of turbulence generated before the nozzle was reduced to a minimum and the turbulent Schmidt number was adjusted to simulated the total / overall effect of the turbulence.

$$
k=\frac{3}{2}\left(u_{a v} I\right)^{2}
$$


$\varepsilon=\frac{k^{3 / 2}}{l}$

$l=0.007 D_{H}$

The second order upwind discretization scheme was used for momentum, turbulent kinetic energy and turbulent energy dissipation rate while QUICK scheme was used for volume fraction. Phase coupled SIMPLE scheme was used for the pressure-velocity coupling. A relaxation factor of 0.3 and 0.05 was used for the pressure and momentum respectively, while a factor of 0.1 was used for turbulent kinetic energy, turbulent energy dissipation rate and volume fraction. Far greater dispersion and higher gas induction was seen in the solution calculated using the first order upwind discretization schemes. This is due to the fact that numerical diffusion is higher in first order schemes as compared to the second order schemes. The solution was initialized by taking the ejector geometry completely filled with stagnant secondary fluid/air. The solution was iterated until convergence was achieved. The first criterion for convergence is the gas flow rate from the secondary inlet becomes constant and the second is that the residuals for each equation was well below $10^{-5}$.

To minimize numerical error grid independence studies were performed using two structured quad grid of size $1 \mathrm{x}(\sim 100000)$ and $2 \mathrm{x}(\sim 200000)$ respectively. The image of the overall 1x grid and blown up sections for nozzle and throat can be seen in Figure 2. Simulations were performed using each grid and it was found that the maximum percent difference between $1 \mathrm{x}$ and $2 \mathrm{x}$ grid size was less than $2 \%$. As this error is much less than the error of experimental measurement ( 20-25\%) 1x grid was selected for all the further studies. The maximum aspect ratio of the $2 \mathrm{D}$ mesh was approximately 2.5 while the minimum orthogonal quality of the mesh was greater than 0.9. These mesh parameters are acceptable according to general and Ansys Fluent guidelines. As discussed in section 1 gas induction and phase mixing is a bulk phenomenon, while the flow is bounded and the constriction at the mixing chamber does play an important flow. There is no phenomenon that is occurring specifically at the wall in this system. Hence, standard wall function ${ }^{[22]}$ is apt for simulating the walls of the ejector. The $y+$ value in the nozzle section was found to be between 60 and 90 while at the mixing tube wall was found to be around $30^{[18]}$. As Ske model is used to simulate the turbulence the $y+$ values at these important boundaries are acceptable. All simulations were performed using the commercially available CFD software FLUENT 16.0. 


\section{Results and discussion}

Figure 3 compares the profile of volume fraction of liquid in the ejector for both with and without the turbulent dispersion model implementation. In the first contour plot where the model is implemented without turbulent dispersion force the volume fraction contour plots show a completely stratified flow within the ejector. This model implementation is taken from Yadav ${ }^{[15]}$. While mixture model was used by Kandakure ${ }^{[12]}$ and Kim et al. ${ }^{[13]}$ the volume fraction contour plots look the same as that of Yadav ${ }^{[15]}$. The flow was completely stratified and there is absolutely no mixing of the phases. The behaviour of gas and liquid is such that the liquid acts as a downward moving wall and the momentum is transferred to the surrounding gas due to the momentum exchange terms causing the gas to flow. The change in gas induction is solely caused by the interfacial momentum exchange term (drag force; discussed in section 2) which in turn depends upon the bubble diameter. The water phase remains segregated and does not mix in radial direction. The drag force, as seen in Equation (8), acts in a direction which is parallel to the axis and has no significant component in the radial direction. Hence, the solution shown in the first contour plot of Figure 3 is consistent with the model formulation. This suggests that there must be a missing piece in the description of flow physics and which must be incorporated in order to get a mixed velocity as well as phase profiles in the ejector.

Furthermore, these results appear to be counter intuitive to all the experimental studies that report phase mixing in the jet ejector. The experimental studies by Witte, Dirix and Cramer focus fundamentally on the high mass transfer coefficient due to the phase mixing. These experimental results were not even qualitatively reflected in the CFD results without using the turbulent dispersion model. On the other hand, the simulation results using the turbulent dispersion model, as seen from Figure 3, the fluid gets mixed very quickly and then flows along the axis as a two-phase mixture. It is also visible that the mixing between the phases is significantly improved and for the most part the volume fractions of the dispersed phase is 0.40.6 along the axis of the ejector. The results shown in Figure 3 indicate, it is essential to include turbulence dispersion to simulate gas-liquid ejector flow. The results are consistent with the experimental observations, considering gas induction as a phenomenon and the description of the phase profile and the interphase mixing observed in the ejector.

The formation of the jet cone is shown in the velocity contour plot in Figure 4. It is to be noted that the jet expands rapidly in the initial phase and keeps on expanding till the end of mixing tube. However, there is no expansion in the velocity and phase profile of the water in 
the diffuser section. This is primarily observed because the turbulent kinetic energy generated in the liquid nozzle has been dissipated along the length of ejector and is not high enough to cause further expansion. The water velocity profile calculated without the turbulent dispersion model shows no jet cone flows down as there is no momentum drive in the radial direction. Radial motion is observed when the turbulent dissipation force is implemented, the jet expands outwardly in the radial direction. It should be noted that as the diameter of the jet increased about $20 \%$ in the initial stages, as seen in Figure 7 and 8. Yet, there is almost no change in the velocity. This may be attributed to the fact that the surrounding gas is flowing as one with the jet. In other words, the surrounding gas has been induced into the jet and has become a part of the jet itself. Hence, the very nature of the gas induction is captured in after implementing the turbulent dispersion model. Similar phenomenon is seen in the mixing tube section of the ejector where the liquid velocity profiles are similar near the axis of the ejector while having very different phase profiles. This phenomenon can be explained by the fact that the overall momentum of the gas liquid mixture is governed solely by the addition of momentum at the liquid nozzle as seen in Figure 7. Since, a jet ejector is considered as a closed system, there is no source of momentum or energy except for the liquid nozzle. This liquid phase momentum is used to pull the surrounding gas into a coaxial flow and to compensate for the losses at the wall boundaries. Hence, at equal gas induction values the overall velocity of the mixture phase is expected to be similar even when the phase profiles are significantly different.

Figure 5 shows the comparison between the pressure profiles in the venturi part of the ejector calculated with and without the turbulence dispersion model. If the turbulent dispersion is not implemented the pressure changes across the ejector are less $-0.2 \mathrm{kPag}$. Whereas, with the turbulent dispersion model the lowest pressure goes down to less than $-2 \mathrm{kPag}$. As seen in Figure 5, when the overall dispersion coefficient is increased the lowest pressure goes down up to a certain limit. As seen in Figure 3 when turbulence dispersion model is not implemented the gas flow resembles the flow over a moving wall. This flow passes through the mixing tube and as the density of a gas is approximately one thousandth of the liquid negligible pressure drop is seen in the mixing tube. As the turbulent dispersion model is implemented the waterair mixture jet takes up a lot of volume in the mixing chamber and resulting in change in the flow profile and increase in the pressure drop in the tube. However, after a certain value of $\sigma$ when the phase profile develops so as both the phases disperse through the mixing tube radially the minimum pressure in the tube does not drop any further. Furthermore, the change in the rest of the profile depends upon the dispersion at the entry point of the mixing tube, at higher 
dispersion there may be a bump in the pressure corresponding to the pressure drop of the tube. This is akin to the "bump" described by Witte as a discontinuity, it may be argued that this increase in pressure is caused in order to overcome the pressure drop in the mixing tube. Furthermore, this bump is not seen in the simulations without turbulent dispersion force, hence it may be stated that the bump in pressure is a result of jet dispersion and the flow of two phase jet into a constriction. It may also be said that at lower $\sigma$ the gas induced in the jet envelope is higher but also the pressure drop in the venturi section is higher which compensates for the increased gas induction.

As shown in Equation (7) the dispersion source term or the dispersion of jet has an inverse relation with the turbulent Schmidt ( $\sigma)$. Which is to say the lower is the value of $\sigma$ the higher is the dispersion observed in the jet. This can be seen in Figure 6 where the volume fraction contours for water are shown for 3 different values of $\sigma$. It is evident that as $\sigma$ decreases the jet breakup length, the length at which gas penetrates to the axis of the jet, decreases and there is more mixing and greater jet diameter along the axis of the ejector. This dispersion of the jet diameter can be seen in Figure 7 where the radial profiles of the jet are shown at various distance from the nozzle outlet. As can be seen from the figure, the jet overall diameter increases as the turbulent Schmidt number is lowered. It is to be noted that if the turbulent dispersion force is not implemented the water phase has no tendency to disperse or diffuse into the surrounding air. Furthermore, similar turbulent dispersion affects can be seen in the Figure 8 where the difference in the phase profiles are further pronounced inside the mixing tube where, as experimentally observed the phase mixing is completed.

The impact of mixing coefficient is very significant on not only the gas induction but also the phase and velocity profiles of flow within the ejector. As the turbulent Schmidt number $\sigma_{\mathrm{k}}$ is decrease the overall dispersion coefficient increases and there is an increase in the jet envelope and the mixing of the jet gets more pronounced. At the same time the increase in the jet envelope causes more gas to flow with the liquid resulting in higher gas induction as shown in Figure 9. The jet diameter in the mixing tube also increases with the decrease in turbulent Schmidt number. The jet diameter is equivalent to the mixing tube diameter at the end of mixing tube at $\sigma_{\mathrm{k}}=0.3$. As it is observed experimentally that till the end of mixing tube the overall mix jet's diameter is equal to mixing tube diameter. Hence, 0.3 is the value selected for further simulations. 
This model described by Yadav was used to fit gas induction in jet ejectors. The simulated gas induction results were compared to experimental values ${ }^{[5]}$ as shown in Figure 10. As seen in the figure the gas induction agrees with the experimental values at the $0.0015 \mathrm{~m}$ or $1.5 \mathrm{~mm}$ gas bubble diameter. This observation is very much in line with the drag formulation shown in Equation (9). Drag force is the momentum exchange term between the phases and it decreases with the increase in bubble diameter. As drag is the only source of momentum transfer between water or the primary fluid and air the secondary fluid any decrease in this term will decrease the gas velocity in the jet envelope and hence decrease in gas induction. However, it should be noted that the difference between the calculated gas induction at 0.8 and $1.5 \mathrm{~mm}$ bubble diameters is within $10 \%$. Which is to say that the sensitivity of gas induction with respect to bubble diameter is not very high and since the model predictions are within $10 \%$ lower than experimental accuracy of $25 \%$ any value of bubble diameter might give an acceptable value. This is a vast improvement from the previous work using mixture model and a percentage based slip formulation using which the results varied drastically ${ }^{[12]}$.

The relationship of bubble diameter of the gas induction is an inverse one. When bubble diameter is increased the interphase drag force decrease increasing the slip velocity between the phases. The second relationship seen in Figure 9 is that as the turbulent Schmidt number decreases, increase the turbulent dispersion, and further increasing the jet envelop, an increase in gas induction was observed in the simulations. In Figure 11, like the observation in Figure 10 , gas induction decreases with the increase in the bubble diameter. However, the fitted value of gas induction with turbulent dispersion is lower than the fitted value without. This can be attributed to the fact that the pressure drop in the mixing tube is higher in the case of turbulent dispersion as seen in Figure 5. This counter balance puts a cap on gas induction resulting the values we see in Figure 11. Further analysis suggests that the sensitivity of gas induction on bubble diameter is not very high. The value of gas induction varies around $15 \%$ when bubble diameter is varied from $0.01 \mathrm{~mm}$ to $1 \mathrm{~mm}$ while there is no impact on the phase profile. It should be noted that while using the revised model the value of bubble diameter at which the simulated gas induction fits the experimental gas induction is roughly (1/40) times the diameter of the mixing tube which is a better estimate as compared to previous results where the value was approximately (1/10). While gas induction will rise as the expanding jet cone and which is compensated higher pressure drop in the mixing tube. Also the figure shows that decreasing the bubble diameter beyond $0.2 \mathrm{~mm}$ there is no significant change in the gas induction. 


\section{Conclusions}

The present work attempts to understand the phase profile in a gas-liquid ejector and asserts that the experimental evidence of phase dispersion or jet breaking is because of the turbulence of the jet. It was found that solving the turbulent dispersion does cause the phase profile in an ejector to be more dispersed and any simulation without this force will have a near perfect stratified flow. The diameter of jet and the overall dispersion was found to be dependent upon the inverse of the turbulent Schmidt number. The jet dispersion was found to be independent of bubble diameter of the secondary phase. As expected gas induction decreased with the increase in the bubble diameter of gas. However, gas induction was not found to be very sensitive w.r.t. bubble diameter as the change in gas induction was only $10-15 \%$ over a 100 fold increase in the bubble diameter. In addition to the phase profile of the primary and the dispersed phase, it was seen that the minimum pressure in the venturi tube, gas induction and the momentum imparted by the liquid nozzle or the power of the nozzle are intricately connected. The study indicated that the minimum pressure in the mixing tube decreased with the increase in effective turbulent dispersion keeping the overall velocity relatively same.

The corrected phase profiles are useful in the development of mass transfer CFD models. As seen in the results section a completely stratified flow solution might not capture the overall and the local effects impacting the mass transfer. On the other hand the turbulence dispersion model predicts the phenomenon of phase mixing correctly with realistic bubble diameter values. Hence, it can be concluded that turbulence dispersion is the root cause of the phase dispersion and can be modelled using RANS framework diffusion terms using a coefficient consisting of turbulent viscosity and a constant. The bubble diameter and the coefficient plays an important role in both the phase profile as well as the gas induction in a gas ejector. Finally, it can be said that the dispersion model at the very least qualitatively predicts the basic flow patterns in an ejector and can be extended to mass transfer / reactor level models.

\section{Future Work}

The mathematical model developed in this paper may be further utilized for understanding the dynamics of the jet and the nature of gas - liquid interaction in an ejector. In the future work the authors would like to suggest 3 things. First, experimental data on the system, specific to anemometric velocity measurements and measurement of bubble diameter 
using imagery or other relevant techniques. Secondly, through experiments an in-depth understanding the anisotropic behaviour of the two-phase jet. Specifically, a detailed analysis of how the mixing tube impacts the gas - liquid interaction in the bulk of the two phsae jet and how the turbulent behaviour changes due to the presence of the throat/obstruction/mixing tube in the path of the jet. Finally, the modification of the current framework to incorporate the experimental findings using relevant source terms and modifications of the dispersion coefficients.

\section{Symbols and Notations}




\begin{tabular}{|c|c|c|c|}
\hline Sr. \# & Symbol & Quantity & Unit \\
\hline 1 & $u$ & Velocity & $\mathrm{m} / \mathrm{s}$ \\
\hline 2 & $U$ & Average Velocity & $\mathrm{m} / \mathrm{s}$ \\
\hline 3 & $\rho$ & Density & $\mathrm{kg} / \mathrm{m}^{3}$ \\
\hline 4 & $\alpha$ & Volume fraction & - \\
\hline 5 & S & Source & $\mathrm{kg} / \mathrm{sm}^{3}$ \\
\hline 6 & $\mu$ & Viscosity & Pa.s \\
\hline 7 & $\sigma$ & Turbulent Schmidt number & - \\
\hline 8 & $p$ & Pressure & $P a$ \\
\hline 9 & $d_{p}$ & Diameter of $\mathrm{p}^{\text {th }}$ phase & $m$ \\
\hline 10 & $C_{D}$ & Drag coefficient & - \\
\hline 11 & $R e$ & Reynolds Number & - \\
\hline 12 & $\vec{g}$ & Directional Gravity & $m / s^{2}$ \\
\hline 13 & $x$ & Spatial co-ordinates & $m$ \\
\hline 14 & $k$ & Turbulent kinetic energy & $m^{2} / s^{2}$ \\
\hline 15 & $\overline{\bar{\tau}}$ & Stress Tensor & $N / m^{2}$ \\
\hline 16 & $G$ & Turbulence Generation term & $m^{2} / s$ \\
\hline 17 & $\varepsilon$ & Turbulent energy dissipation rate & $m^{2} / s^{3}$ \\
\hline 18 & $u^{\prime}$ & Fluctuating velocity & $\mathrm{m} / \mathrm{s}$ \\
\hline 20 & $\alpha^{\prime}$ & Fluctuating Volume fraction & - \\
\hline 21 & $I$ & Turbulent Intensity & $\%$ \\
\hline 22 & $D_{H}$ & Hydraulic diameter & $m$ \\
\hline
\end{tabular}


23

24

25

$v$

$$
D_{T}
$$

Throat diameter

Turbulent Length scale

$m$

Kinematic Viscosity

$\operatorname{Pas}$

Subscript/Superscript

$\mathrm{Sr}$ \#

Subscript/Superscript Quantity

1

$i, j$

Direction

vectors/Species

2

$m$

mixture

3

$k, p, q$

Phase ID

4

Av

Average

5

$\mathrm{t}$

Turbulent

6

Av

Average 


\section{References}

1. Delas, A. Steam ejector with cooled diffuser. US patent 1,529,774 A. 1925.

2. Cramers, P.H.M.R.; Beenackers, A.A.C.M. Influence of ejector configuration, scale and the gas density on the mass transfer characteristics of gas-liquid ejectors. Chem. Eng. J.2001, 82, 131.

3. Dirix, C.A.M.C. \& van der Wiele, K. Mass transfer in jet loop reactors. Chem. Eng. Sci.1990, 42, 2333.

4. Pangarkar, V.G. Design of Multiphase Reactors, $1^{\text {st }}$ Edition; John Wiley and Sons: Hoboken, New Jersey, 2015; pp 429.

5. Bhutada, S. R.; Pangarkar, V. G. Gas induction and hold-up characteristics of liquid jet loop reactors. Chem. Eng. Comm. 1987, 61, 239.

6. Zahradnik, J.; Fialova, M.; Linek, V., Subjuke, J., Reznickova, J. Dispersion efficiency of ejector type gas distributors in different operating model. Chem. Eng. Sci. 1997, $52,4499$.

7. Witte, J.H. Mixing shocks in two-phase flow. J. Fluid Mech. 1969, 36, 639.

8. Utomo, T.; Jin, Z.; Rahman, M.; Jeong, H.; Chung, H. Investigation on hydrodynamics and mass transfer characteristics of a gas-liquid ejector using three dimensional CFD modelling. J. Mech. Sci. Tech.2008, 22, 1821.

9. Ben Brahim, A.; Prevost, M. \& Bugarel, R. Momentum transfer in a vertical down flow liquid jet ejector: Case of self-gas aspiration and emulsion flow. J. Multiphase Flow. 1984, 10, 79.

10. Bin, A. K. Gas entrainment by plunging liquid jets. Chem. Eng. Sci.1993, 48, 3585.

11. Kitamura, Y.; Takahashi, T. Influence of nozzle length on breakup of jet. Proceedings of $1^{\text {st }}$ International Conference on Liquid Atomization and Spray Systems. 1978, ICLAS, Tokyo.

12. Kandakure, M. T.; Gaikar, V. G.; Patwardhan, A. W. Hydrodynamic aspects of ejectors. Chem. Eng. Sci. 2005, 60, 6391.

13. Kim, M. I.; Kim, O. S.; Lee, D. H.; Kim, S. D. Numerical and Experimental investigations of gas-liquid dispersion in an ejector. Chem. Eng. Sci. 2007, 62, 7133.

14. Manninen, M.; Taivassalo, V., Kallio, S. On the mixture model for multiphase flow. VTT Publications. 1996, 288, 67. 
15. Yadav, R. L.; Patwardhan, A. W. Design aspects of ejectors: Effect of suction chamber geometry. Chem. Eng. Sci. 2008, 63, 3886.

16. Shinijo, J.; Umemura, A. Simulation of liquid jet primary breakup: Dynamics of ligament and droplet formation. Int. J. Multiphase Flow. 2010, 36, 513.

17. Xiao, F.; Dianat, M.; McGuirk, J. J. LES simulation of turbulent primary breakup in turbulent coaxial flow. Int. J. Multiphase Flow. 2014, 60, 103.

18. Ranade, V.V. Computational Flow Modelling for Chemical Reactor Engineering, $1^{\text {st }}$ Edition; Academic Press: California, 2002; pp 41.

19. Johansen, S.T. (1988). On the modelling of dispersed two phase flow, PhD thesis, University of Trondheim, Norway

20. Sharma, D.V.; Patwardhan A.W.; Ranade, V.V. Estimation of gas induction in jet loop reactors: Influence of nozzle design. Chemical Engineering Research and Design. 2017, 125, 24.

21. Chanson, H. Hydraulics of aerated flows: qui pro quo? Journal of Hydraulic Research. 2013, 51:3, 222.

22. ANSYS ${ }^{\circledR}$ Fluent, Release 16.0, Fluent Theory Guide, Basic Fluid Flow (1), Turbulence (4), Species Transport and Finite Rate Chemistry (7), ANSYS, Inc.

23. Pope, Stephen. Turbulent Flows. Cambridge University Press, 2000.

24. Ranade, V. V. Modelling of turbulent flow in bubble column reactors. Institute of Chemical Engineers (I Chem E). 1997, 75 (A), 14.

25. Ranade, V. V. and Van den Akker, H. E. A. A computational snap-shot of gas-liquid flow in baffled stirred vessels, Chem Eng Sci. 1994, 49: 5175. 


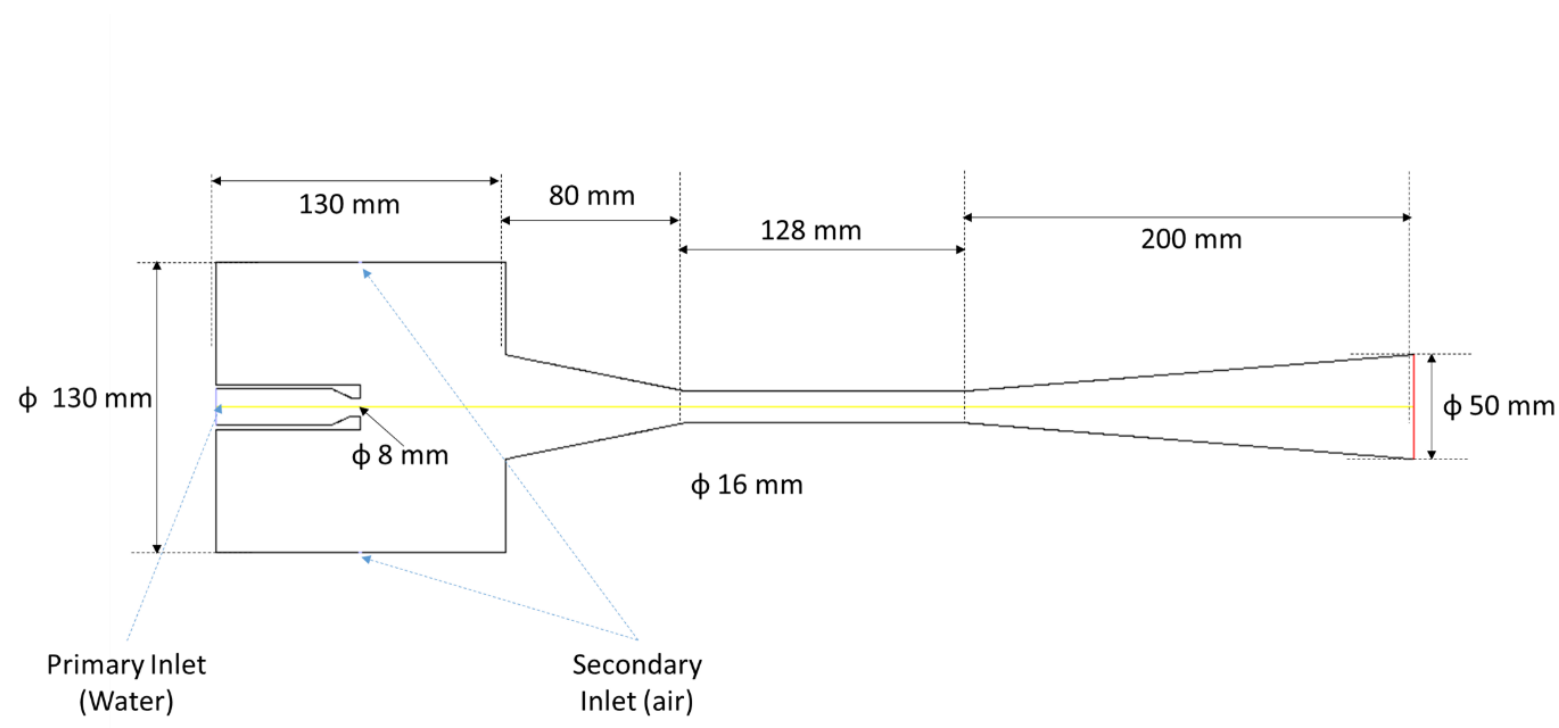

Figure 1: Geometry of the Ejector simulated

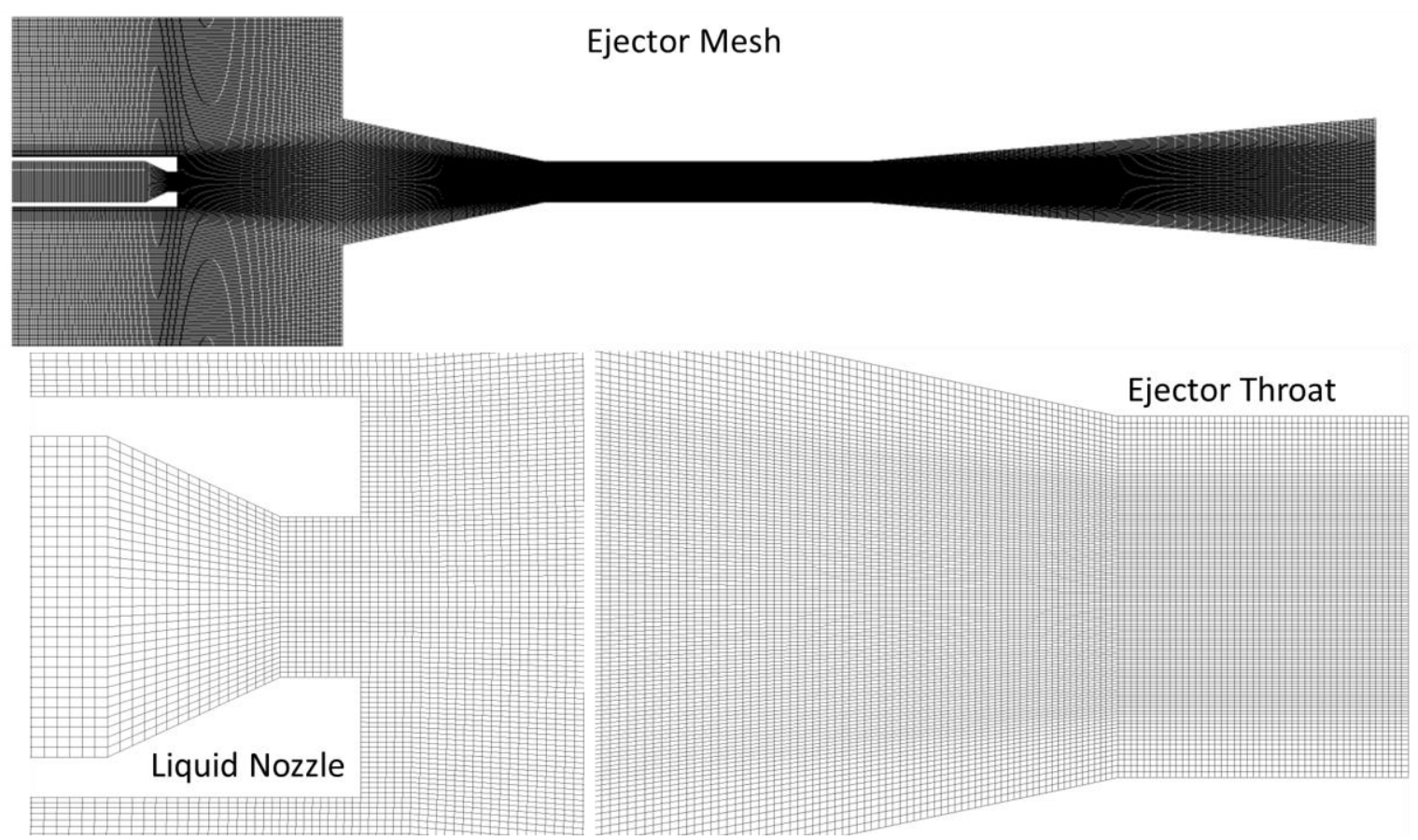

Figure 2: Mesh of Ejector 


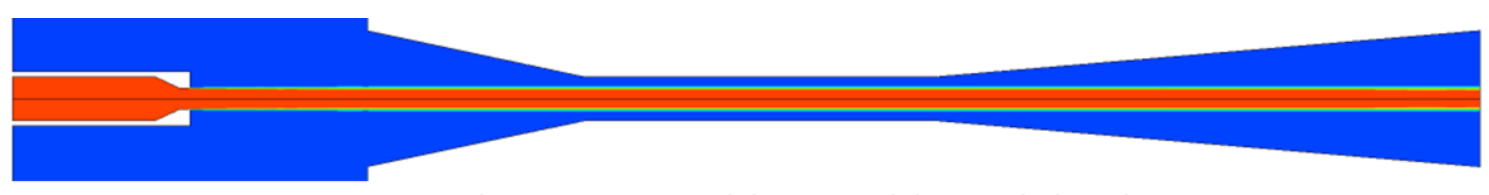

VOF Contour plot @ Mixture Model \& EE Model; no turbulent dispersion

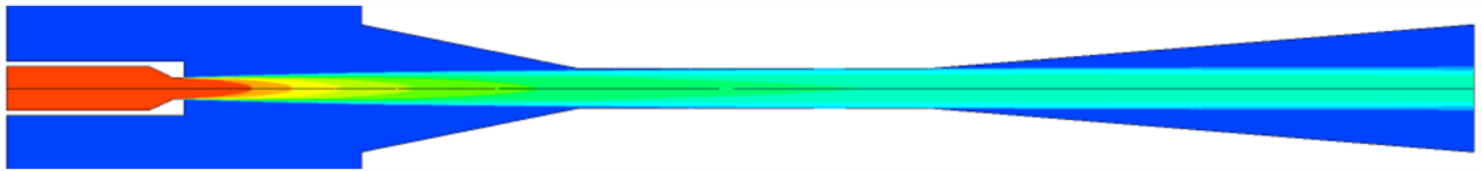

Velocity Contour plot @ EE Model using turbulent dispersion

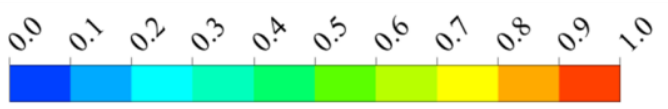

Figure 3: Contour plot for volume fraction of water inside gas-liquid ejector with \& without turbulent dispersion

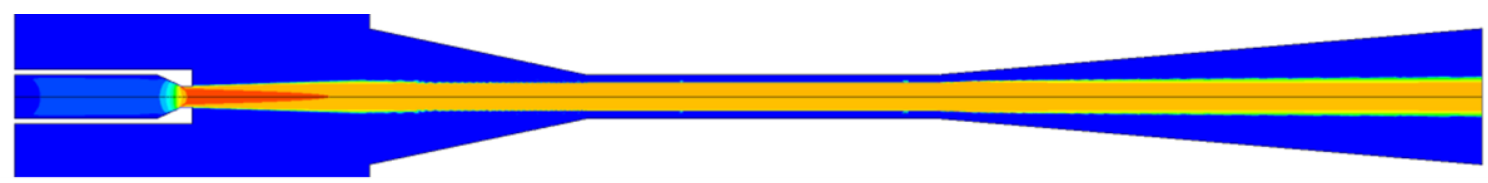

Velocity Contour plot @ Mixture Model \& EE Model; no turbulent dispersion

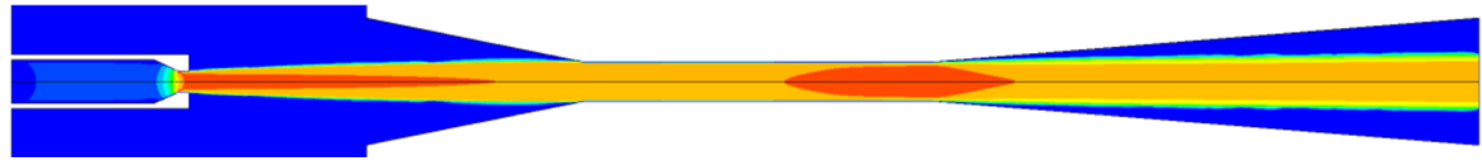

Velocity Contour plot @ EE Model using turbulent dispersion

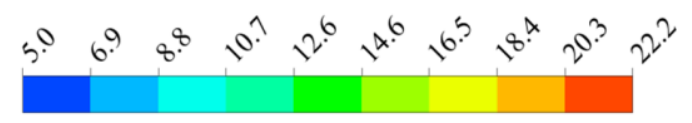

Figure 4: Contour plots for comparison of velocity profiles of water with and without turbulent dispersion model

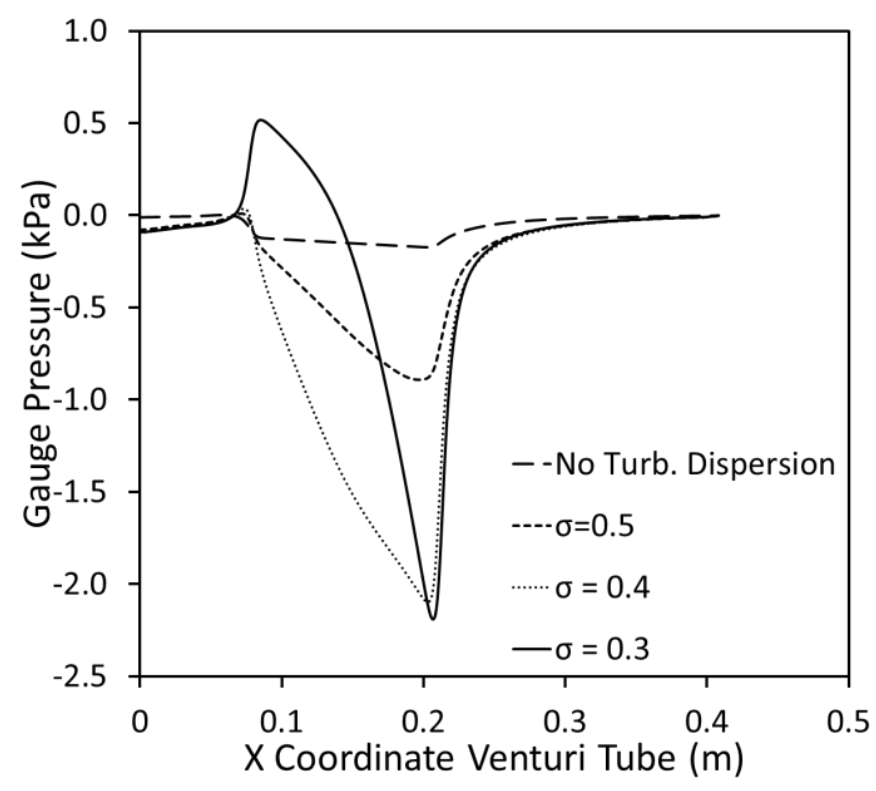


Figure 5: Comparison of pressure along the axis calculated with and without turbulent dispersion model

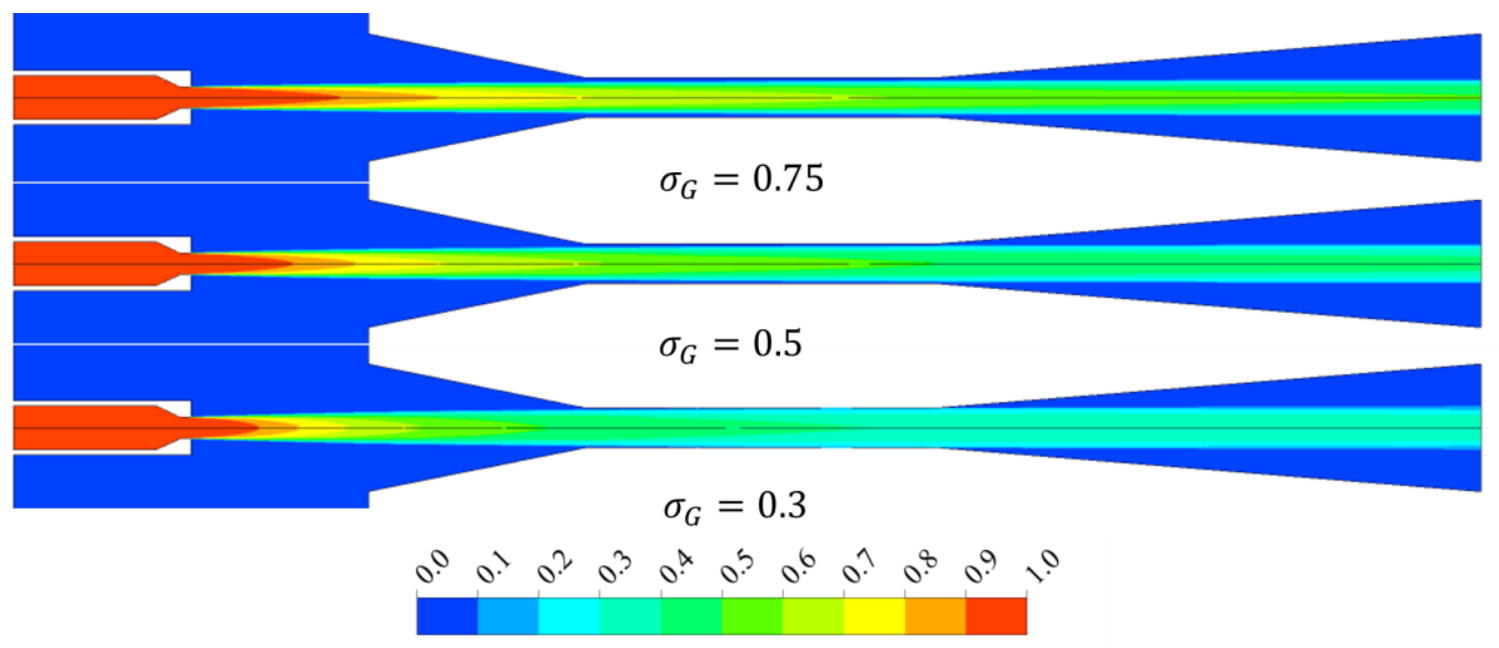

Figure 6: Contour plots of volume fraction of water showing the impact of turbulent Schmidt number on the phase dispersion between air and water 

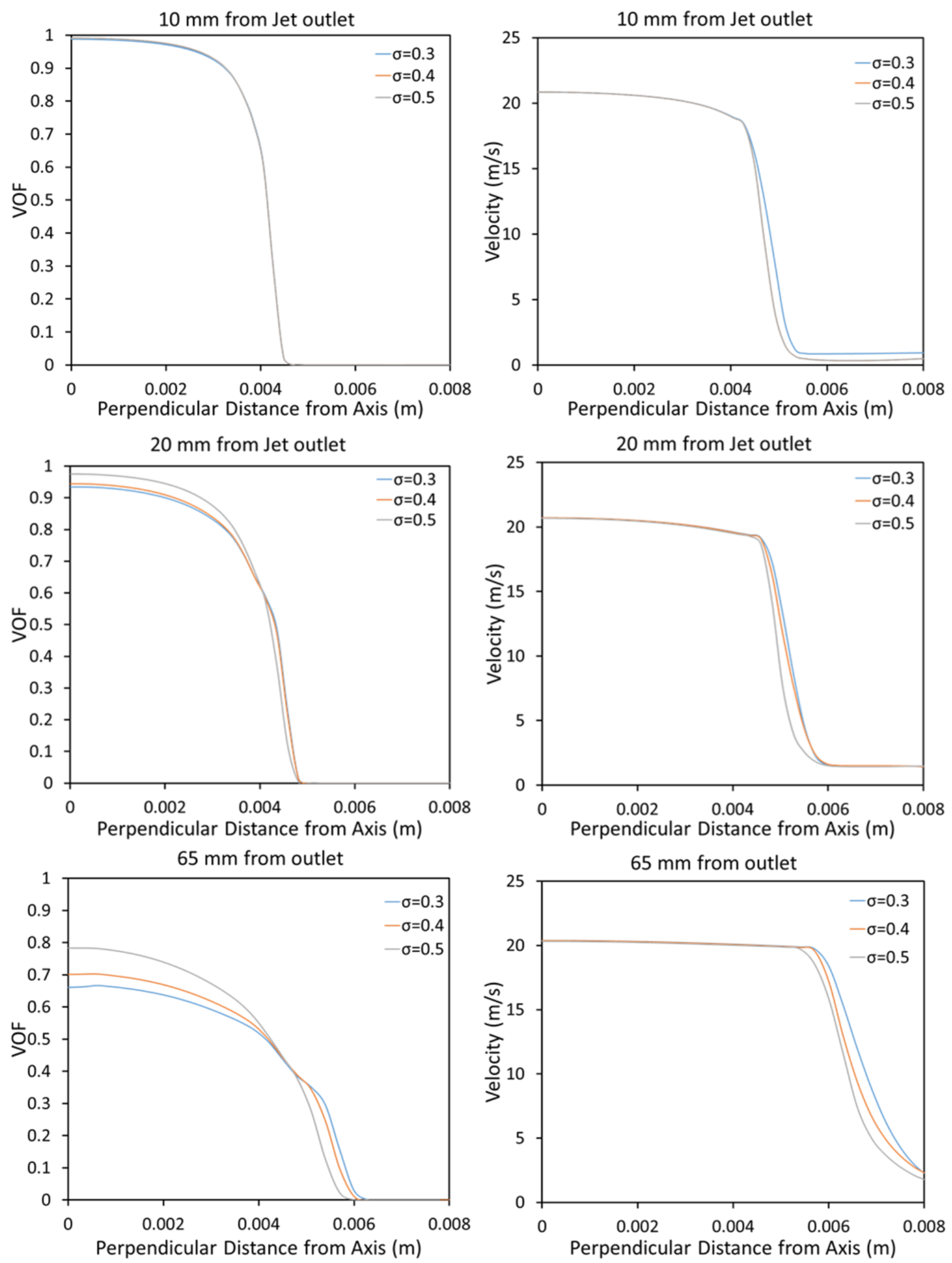

Figure 7: Radial phase and velocity profiles of water at different distances from the nozzle outlet at different values of turbulent Schmidt number $(\sigma)$ 

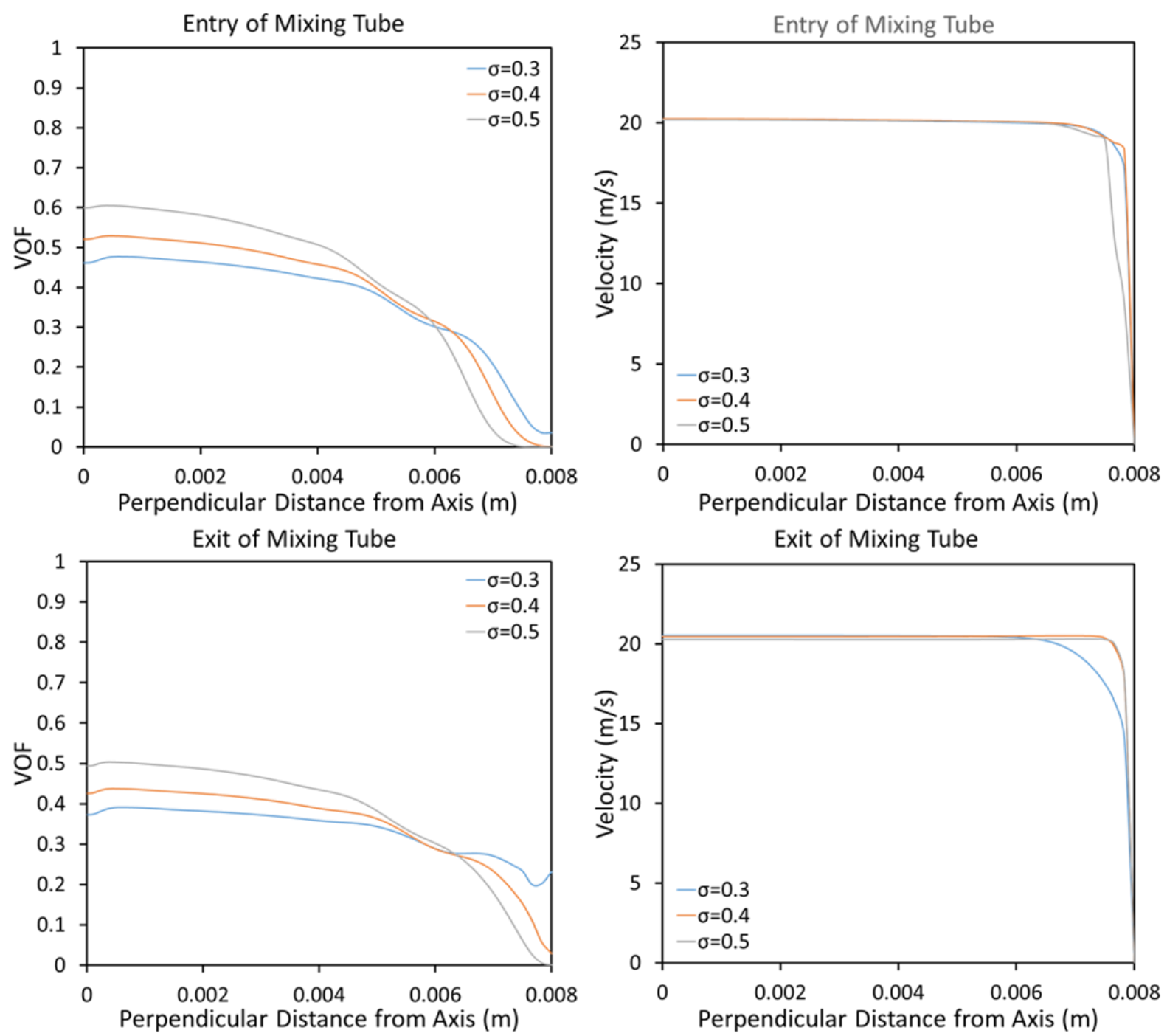

Figure 8: The volume fraction and velocity of water contour plots at the start and end of the mixing tube section of the ejector 


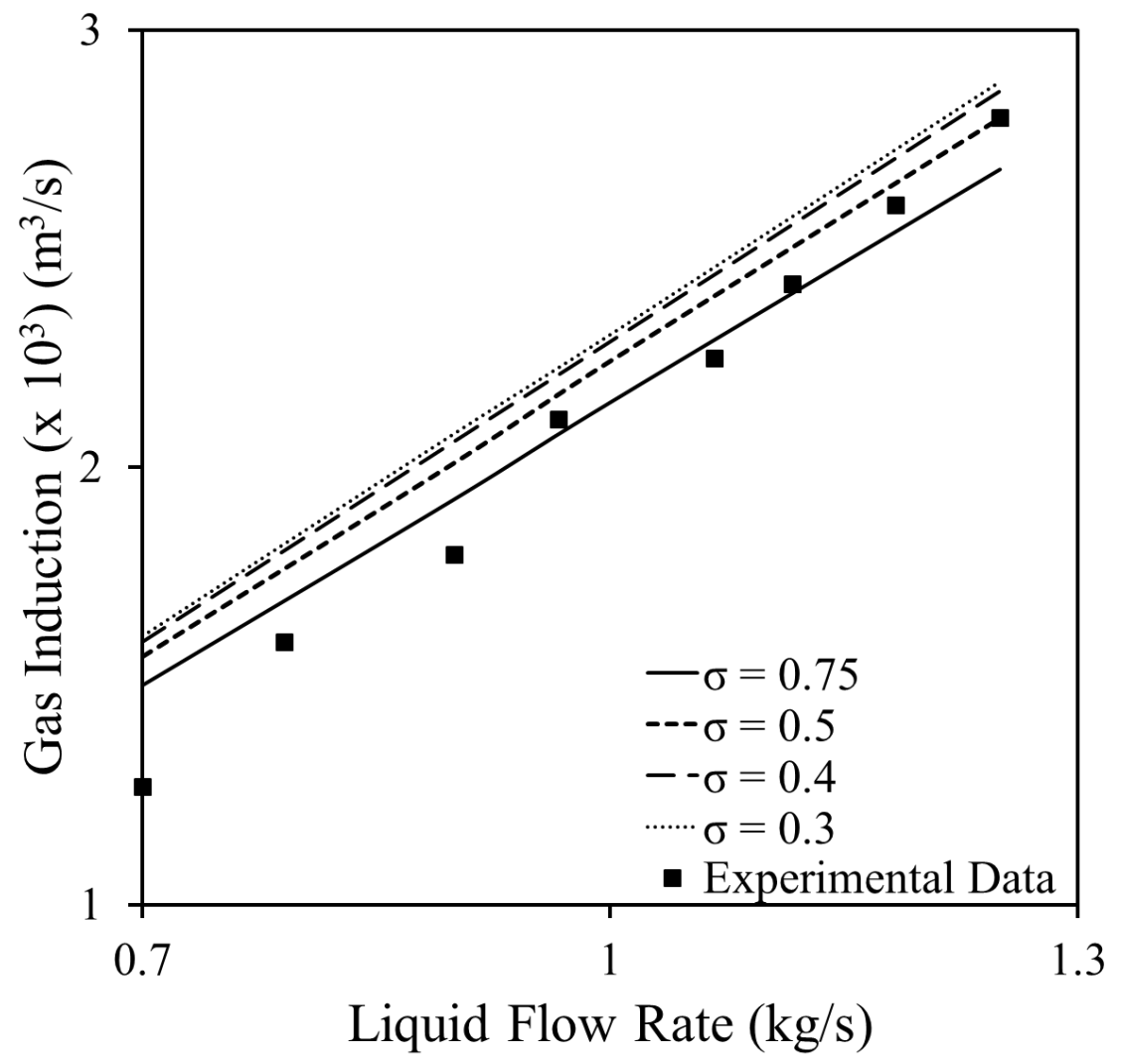

Figure 9: Effect of Turbulent Schmidt number on gas induction

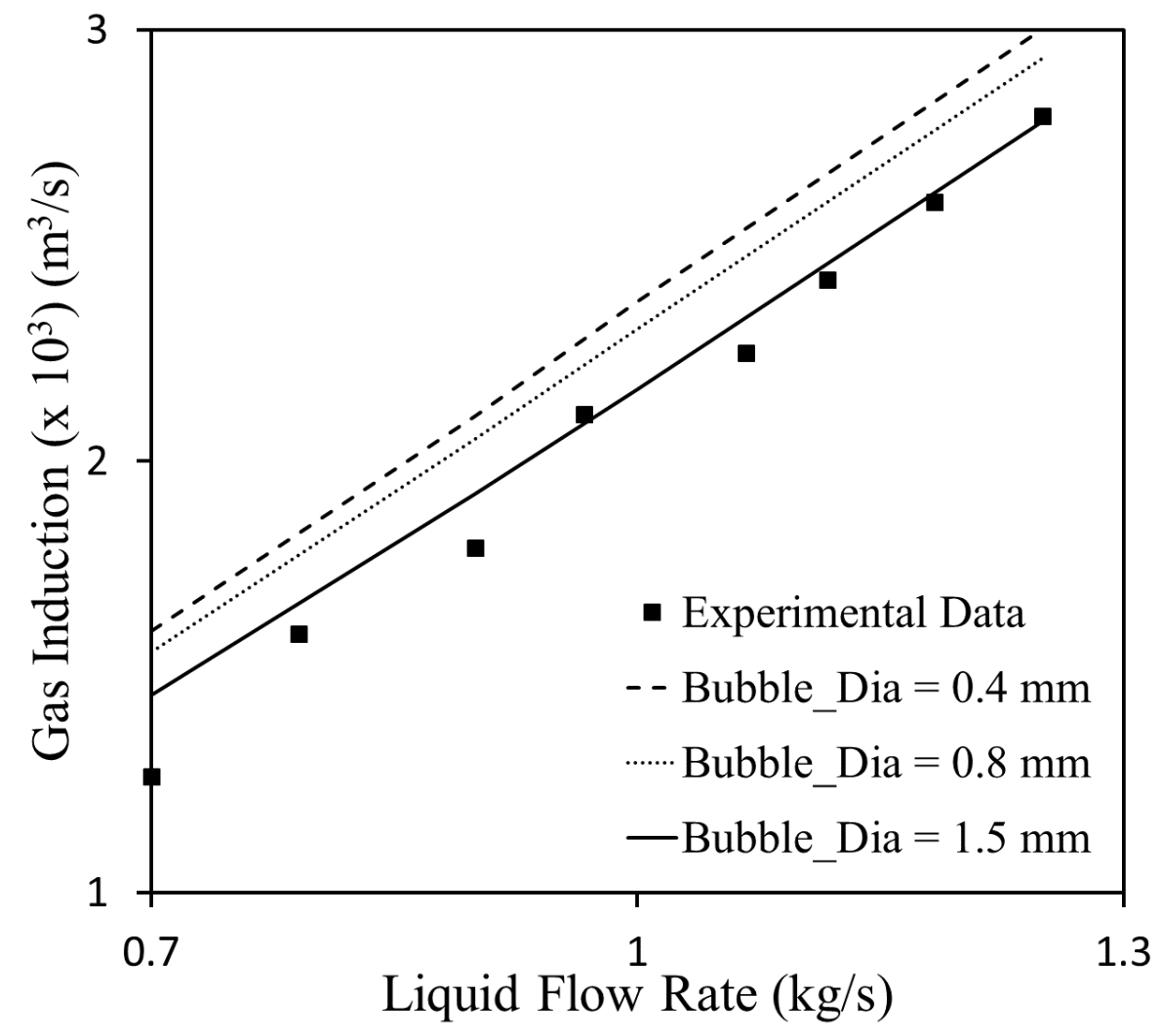


Figure 10: Experimental and simulated gas induction at different bubble diameter; without turbulence dispersion

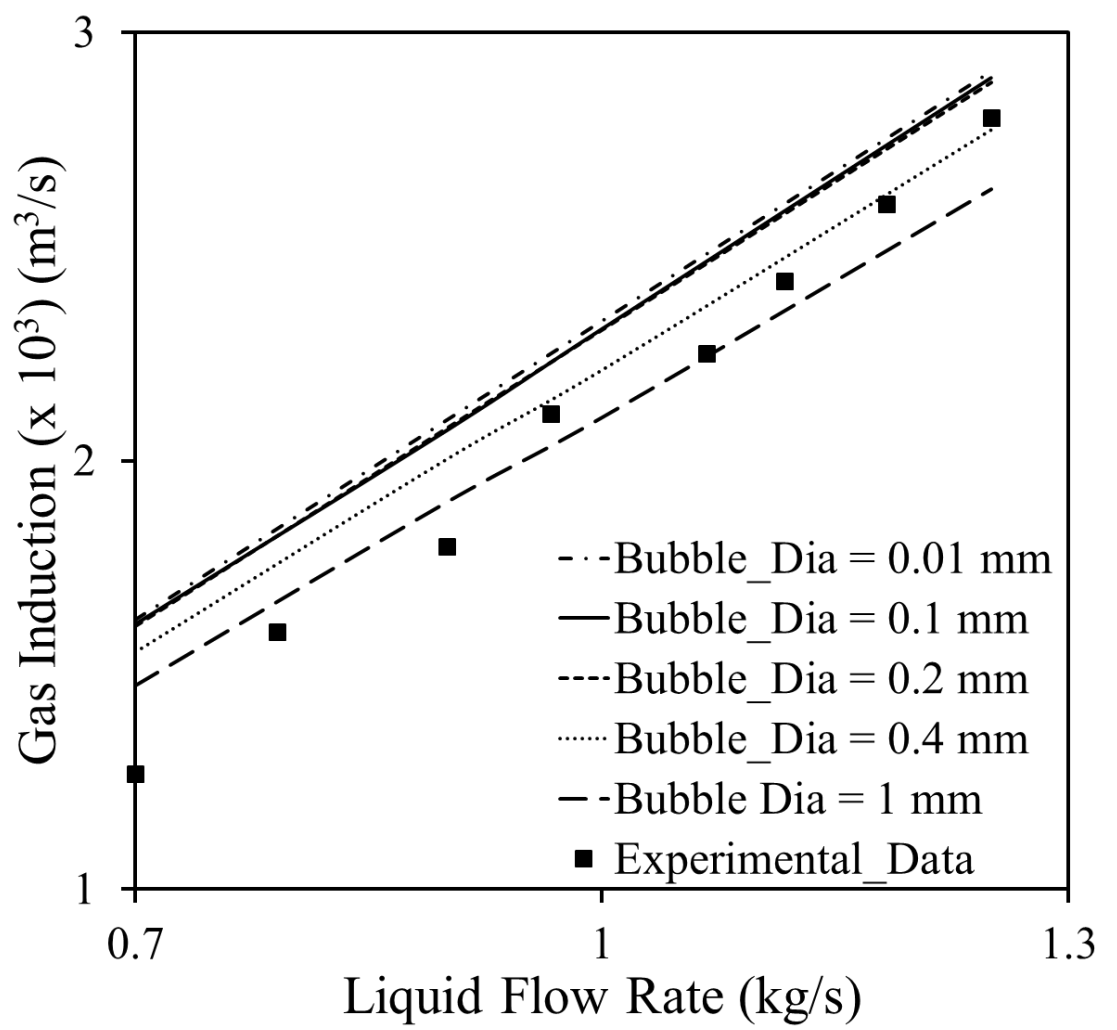

Figure 11: Effect of bubble diameter on gas induction; Model simulated with turbulent dispersion 
Table 1: Boundary Conditions

\begin{tabular}{|c|c|c|}
\hline Boundary of the System & Type of Boundary Condition & Values \\
\hline Primary Inlet & Mass Flow inlet & $0.7-1.25(\mathrm{~kg} / \mathrm{s})$ of water \\
\hline Secondary Inlet & Pressure Inlet & Gauge Pressure $=0 \mathrm{~Pa}$ \\
\hline Outlet & Pressure Outlet & Gauge Pressure $=0 \mathrm{~Pa}$ \\
\hline $\begin{array}{c}\text { Walls of Ejector (Venturi, } \\
\text { Nuction chamber and } \\
\text { Nozzle) }\end{array}$ & Wall & $\begin{array}{c}\text { No slip, adiabatic } \\
\text { Axial Symmetry (2 D } \\
\text { axi-symmetry } \\
\text { simulation) }\end{array}$ \\
\hline Axis of Ejector & Symmetry & \begin{tabular}{c} 
\\
\hline
\end{tabular}
\end{tabular}

Table 2: Closure Models

\begin{tabular}{|c|c|}
\hline Modelled Quantity & Closure Model Used \\
\hline Drag Force & Schiller - Naumann \\
\hline Lift Force & None \\
\hline Virtual Mass Force & None \\
\hline Turbulence & Standard k $-\varepsilon$ \\
\hline
\end{tabular}

